\title{
AU COEUR
}

\section{DE L'ACTUALITÉ}

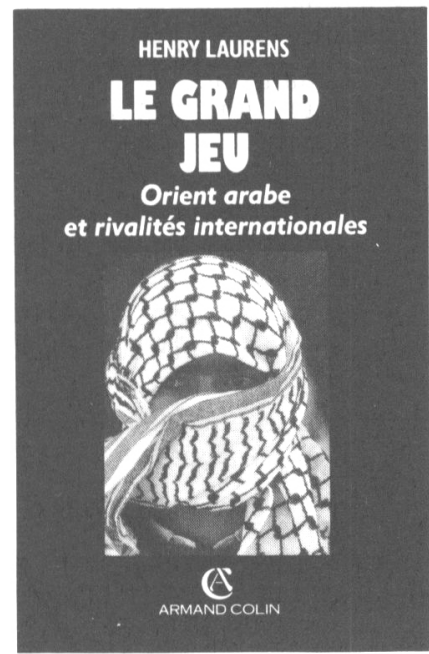

"... Lisez, de la première à la dernière ligne, ce livre définitif sur la question du

Proche-Orient. "

François Granon, TELERAMA

"... précieux si l'on prétend s'interroger sérieusement sur la suite de la guerre du Golfe."

André Laurens, LE MONDE

Collection Histoires/Colin 488 pages, $180 \mathrm{~F}$.

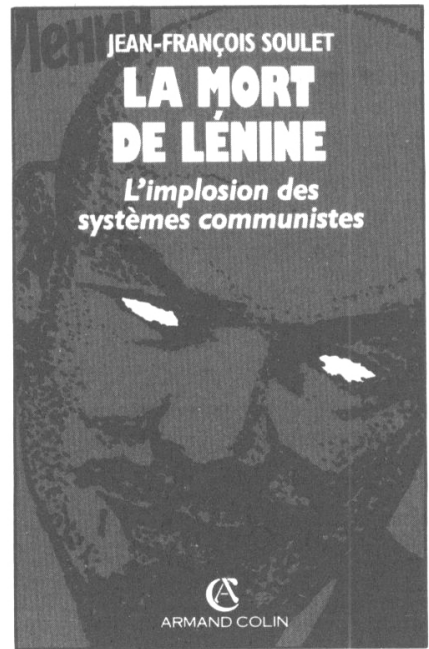

Pourquoi le monde communiste a-t-il basculé?

J.-F. Soulet reconstitue le processus de désagrégation en le replaçant dans son cadre géopolitique le plus large et en procédant à une étude comparée des forces internes et externes. Un ouvrage essentiel pour la compréhension des derniers événements en URSS.

Collection Histoires/Colin 278 pages, $120 \mathrm{~F}$. 


\section{REVUE ÉCONOMIQUE}

Publication bimestrielle coéditée par le Centre national de la recherche scientifique, l'École des hautes études en sciences sociales at la Fondation nationale des sciences politiques

\section{SEPTEMBRE 1991 : VOL. 42 N`5}

P. ROGER

B. WALLISER

M. GermanN

B. GRAND

P. VILIAA

T. N'GuESSAN
Options et complétude des marchés.

Logique épistémique et théorie des jeux.

Le principe de conservation de la matière dans le cadre du modèle d'HARROD-DOMAR.

Les incitations financières au crédit commercial international.

Règles de taux d'intérêt en changes flexibles et en présence de chocs permanents.

Un modèle de comportement bureaucratique de la Banque Centrale : le cas de la BCEAO.

Notes de lecture :

J. CartelIER L'économie politique de F. Quesnay : à propos d'un ouvrage de $\mathrm{G}$. Vaggi.

Ventes et abonnements :

Presses de la Fondation Nationale des Sciences Politiques

27, rue Saint-Guillaume, 75341 Paris Cedex 07

Abonnements 1991 : 6 numéros par an

Rédaction :

\begin{tabular}{llc} 
& France & \multicolumn{1}{c}{ Etranger } \\
Institutions ...... & $610 \mathrm{~F}$ & $660 \mathrm{~F}$ \\
Particuliers ....... & $390 \mathrm{~F}$ & $440 \mathrm{~F}$ \\
Etudiants ......... & $280 \mathrm{~F}$ sur présentation CE
\end{tabular}

Prix au numéro : $110 \mathrm{~F}$

54, Boulevard Raspail, 75006 Paris 


\section{HISTOIRE DE LA PENSÉE HISTORIQUE}

G. Dhoquois

Depuis la naissance du mythe et de l'épopée,

l'histoire selon les prophètes,

à Athènes ou à Rome, celle des légistes,

des militants, des écrivains,

jusqu'aux grands débats contemporains

sur l'anti-historicisme et le structuralisme,

l'ouvrage présente les différentes façons dont

les civilisations ont rendu compte de l'histoire

et de la place de l'homme dans l'histoire.

Dans un souci pédagogique,

l'approche se fait essentiellement par auteur.
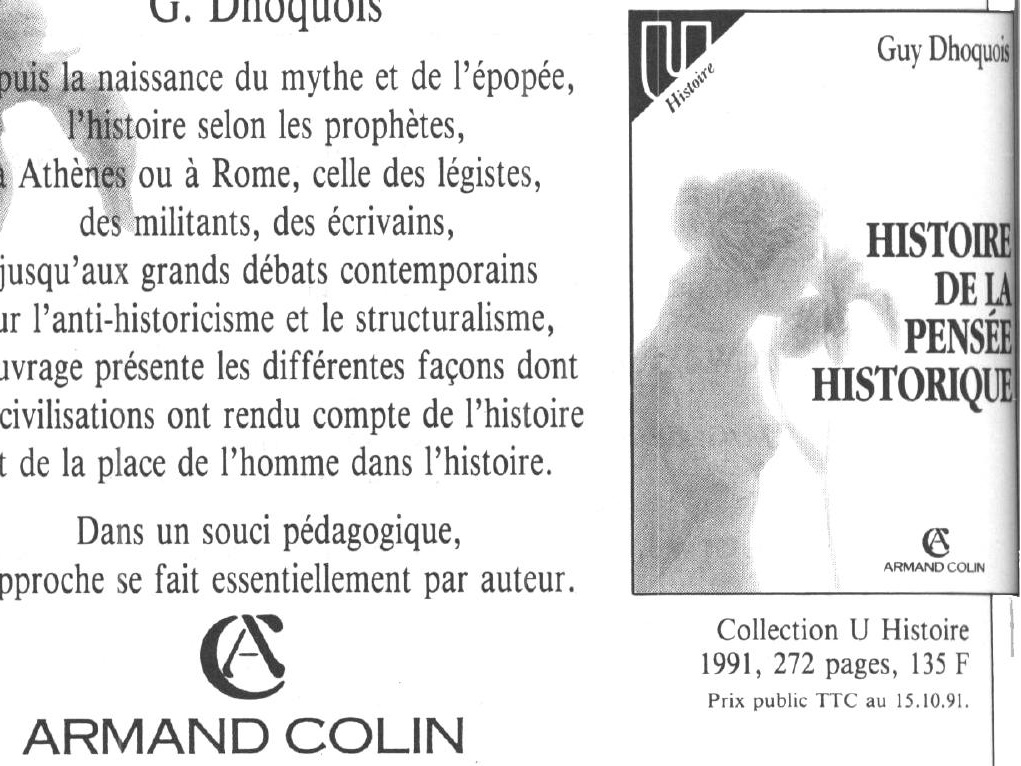

Collection U Histoire 1991, 272 pages, $135 \mathrm{~F}$ Prix public TTC au 15.10.91.

\section{ARMAND COLIN}

\section{O STRATIFICATION ET MOBILITÉ SOCIALE}

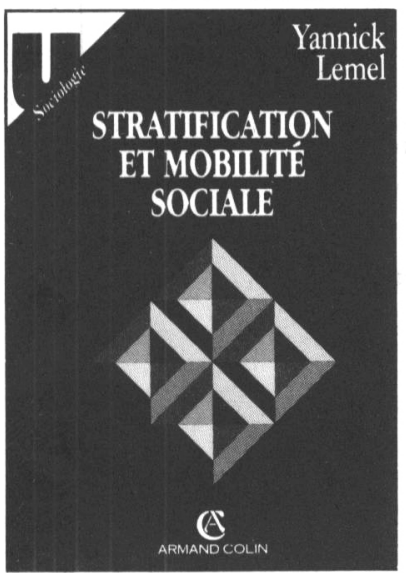

Y.Lemel $\begin{aligned} & \text { Collection } U \text { sociologie } \\ & \text { 1991, } 226 \text { pages, } 148 \mathrm{~F} .\end{aligned}$

L'ouvrage offre une vue d'ensemble des inégalités en France et des formes qu'y prennent la stratification et la mobilité sociale. L'auteur présente les principales orientations théoriques proposées par les sociologues et confronte systématiquement les propositions théoriques avec les données d'observation, notamment statistiques. Il dresse un bilan des travaux et recherches dans le domaine, en indique les limites et la portée.

\section{(A) ARMAND COLIN}




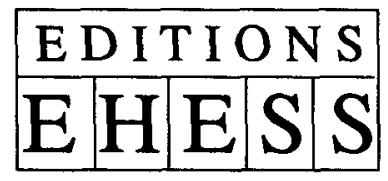

Éditions de l'Éoole des Hautes Etudes en Sciences Sociales

\section{RAYMOND JAMOUS}

\section{La relation frère-sœur Parenté et rites chez les Meo de l'Inde du Nord}

La littérature anthropologique a beaucoup insisté sur les groupes à filiation unilinéaire ou sur l'alliance de mariage, mais rarement sur la spécificité de la relation frère-sœur. Jusqu'à présent, celleci a été réduite soit à la germanité, soit, le plus souvent, à la consanguinité, soit, encore, à une forme de prohibition de l'inceste qui ouvre sur l'échange matrimonial. La nouveauté de cet ouvrage réside dans sa présentation du système de parenté des Meo (communauté musulmane mais aussi caste guerrière de l'Inde du Nord) : la relation frère-sœur y transcende les distinctions entre consanguins et affins, elle se développe non seulement en amont mais aussi en aval du mariage. Pour traduire la spécificité de cette relation, la notion de métagermanité a été forgée.

Cette métagermanité s'exprime, à travers le vocabulaire de parenté, dans la chaîne de deux paires frère-scuur liées par un mariage. Elle se déploie dans les rites des âges de la vie, par la complémentarité entre la tante paternelle (la sœur mariée), officiante principale des cérémonies, et l'oncle maternel (frère de la mère), qui se charge des prestations importantes. La position rituelle de cette soeur marice par rapport à son frère est homologue à celle entre prêtre brahmane et guerrier kshatriya ou encore entre sacrificateur et sacrifiant.

Dans la communauté meo, la relation frère-sœur se formule à la fois comme une relation de l'identité et comme une relation de la différence absolue. D'une part, elle est de l'ordre de la redondance stérile, du mariage impossible; de l'autre, elle produit la vie, plus précisément, un surcroît de vie qui donne sens au principe d'engendrement. Le double mouvement du mariage sépare la sour du frère puis la fait revenir dans la famille de son frère pour assurer la suite des générations. Ainsi se mesure l'importance rituelle de cette relation qui s'inscrit dans la répétition, c'est-à-dire dans une temporalité sans début ni fin.

Directeur de recherche au CNRS, Raymond Jamous a longtemps travaille au Maroc. Il est l' auteur de Honneur et Baraka. Structures sociales traditionnelles dans le Rif, Cambridge University Press - Maison des Sciences de l'Homme, 1981.

Recherches d'histoire et de sciences sociales, 49 1991. 244 pages

$170 \mathrm{~F}$

\section{DIFFUSION \\ CID}

131 bd Saint-Michel, 75005 Paris

Tél : 43.54.47.15 Fax : 43.54.80.73 


\title{
AUX PRESSES DU CNRS
}

\author{
LES CAPITAUX DE L'ISLAM \\ Sous la direction de G. Beaugé \\ Préface de Michel Chatelus
}

L'Islam intervient de manière directe dans le domaine économique, en particulier par la proscription de taux d'intérêt fixe. Depuis une quinzaine d'années, dans des pays à dominante musulmane, la référence croissante d̀ l'slam a entrainé lapparition d'institutions ef de modes de fonctionnement nouveaux.

Après avoir décrit le cadre religieux ef juridique de l'islamisation de l'économie. l'ouvrage analyse les problèmes complexes que pose au système bancaire l'interdiction de l'intérét.

A travers des exemples spécifiques sont successivement envisagés l'investissement, la mobilisation de l'épargne, le prélèvement de l'impőt, les dépenses publiques, le financement du déficit... Une confrontation entre les systèmes occidentaux et islamiques de régulation de l'économie devient des lors possible.

$1990-16 \times 24-276$ pages ISBN 2-87682-045-5

PRIX: $140 \mathrm{~F}$

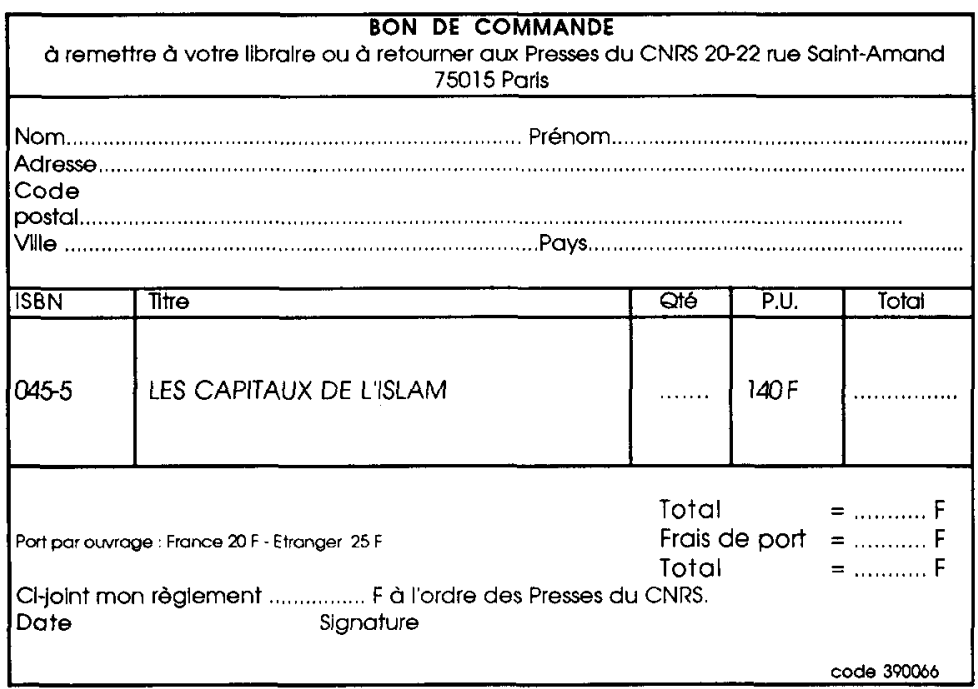

Fax $111453392 \quad 13$

$\overline{I E L I X 200356 F}$

AC. PAAIS $\$ 334311021$

SACAPITAL:4500.000 OEF

C.C.P. PARIS 24795141 


\title{
\begin{tabular}{|l|l|l|l|}
\hline \multicolumn{2}{|c|}{ E D I T I O N S } \\
\hline $\mathrm{E}|\mathrm{H}| \mathrm{E} \mid \mathrm{S}$ & $\mathrm{S}$ \\
\hline
\end{tabular} \\ Editions de l'Ecole des Hautes Etudes en Sciences Sociales \\ PÉRIODES LA CONSTRUCTION DUTEMPS HISTORIQUE
}

\author{
ACTES DU V' COLLOQUE \\ D'HISTOIRE AU PRÉSENT
}

Pour l'historien formé par la tradition occidentale, les périodes délimitent les segments du savoir historique à assimiler. Chacune des «quatre vieilles», ancienne, médiévale, moderne et contemporaine, suppose ses chaires universitaires, ses outils d'érudition et ses débats. La périodisation scolaire engendre ainsi l'organisation sociale et scientifique du métier d'historien. Périodiser rend pensable le chaos des chroniques, ordonne le fil du temps, mais l'opération dissimule mal la quête obstinée d'un sens de l'histoire. L'enjeu de ce cinquième colloque organisé par HISTOIRE AU PRÉSENT est avant tout de mieux comprendre les opérations auxquelles l'historien se livre lorsqu'il s'efforce de rendre le passé (et le présent) intelligible. Si la notion de période se prête particulièrement à cette opération de réveil conceptuel, c'est indéniablement en raison du rôle formateur qu'elle a rempli.

En coédition avec HISTOIRE AU PRÉSENT 1991. 208 pages

$140 \mathrm{~F}$

\section{DIFFUSION}

CID

131 bd Saint-Michel, 75005 Paris

Tél : 43.54.47.15 Fax : 43.54.80.73 


\section{LE MOUVEMENT SOCIAL \\ JUILLET-SEPTEMBRE 1991 NUMÉRO 156}

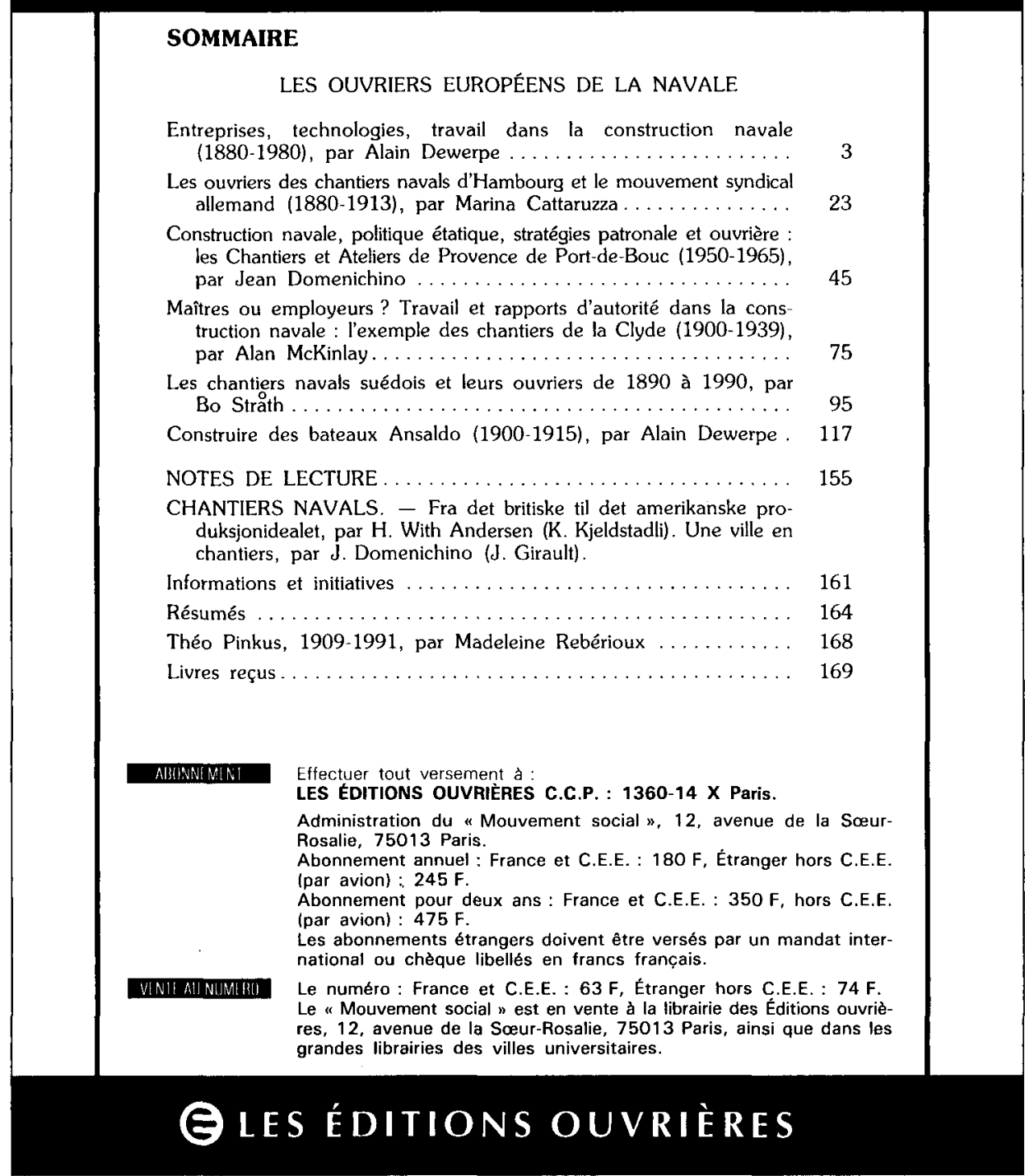




\section{Fondation "Pour la science" Centre international de synthèse}

Directeur: Jean-Claude Perrot
Directeurs adjoints : Michel Blay, Dominique Bourel, Roger Chartier, Ernest Coumet Secrétaire général : Pierre Monzani

\section{Revue \\ de synthèse}

Revue trimestrielle fondée en 1900 par Henri Berr.

Comité de rédaction:

MM. Claude Blanckaert, Dominique Bourel, Eric Brian,

Roger Chartier, Joël Cornette, Ernest Coumet,

HenrieJean Martin, Jacques Merleau-Ponty, Pierre Monzani,

Jean-Claude Perrot, Roshdi Rashed, Daniel Roche.

Comité de lecture:

MM. Guy Beaujouan, Jacques Brunschwig, Paole Casini,

Robert Darnton, Robert Fox, Dominique Julia,

Reinhart Koselleck, Hervé Le Bras, Evrett Mendelsohn,

Stéphane Michaud, Jean Mosconi, Mme Mona Ozouf,

MM. Pierre Pellegrin, Jean-Claude Schmitt,

Pierre Vidal-Naquet, Denis Woronoff.

Secrétaire de rédaction:

Agnès Biard.

\section{AUGUSTE COMTE.}

POLITIQUE ET SCIENCES

Direction-rédaction: Centre International de Synthèse, 12 rue Colbert 75002 Paris - Tél.: 42.97 .50 .68

Administration-abonnements : Editions Albin Michel, 22 rue Huyghens 75014 Paris - Tél. : 42.79.10.00 - CCP Paris $2422223 \mathrm{G}$

Tarif 1991 - Abonnement: France, $300 \mathrm{~F}$; Étranger, $390 \mathrm{~F}$ (Les abonnements partent du $1^{\text {er }}$ janvier.)

Les textes publiés n'engagent que les auteurs. 
Revue

française

d'anthropologie
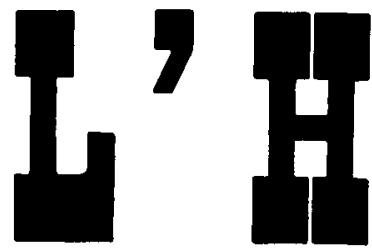

Isabelle COMBËS

Charles ILLOUZ

Sergio Dalla Bernardina

Colette MÉchIN

Jacques Collina-GiRARD octobre
décembre
1991 12
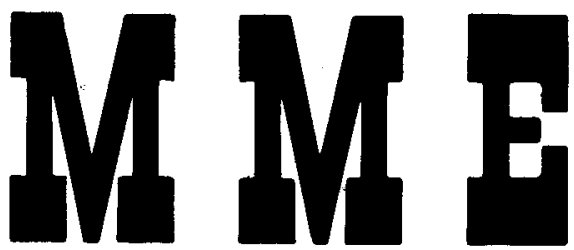

Yaci : mythes et représentations tupi-guarani de la lune

L'Homme aérien aux noces du taro

Une Personne pas tout à fait comme les autres. L'animal et son statut

Les Règles de la bonne mort animale en Europe occidentale

Le Foret à feu : expérimentation contre rumeur scientifique

COMPTES RENDUS - LIVRES REÇUS

Rédaction : Laboratoire d'Anthropologie Sociale, Collège de France

52, rue du Cardinal-Lemoine, 75005 Paris Tél. (1) 44271730

Abonnements: CDR-Centrale des revues, 11, rue Gossin, 92543 Montrouge Cedex.

Tél. (1) 46565266 . Règlement par chèque bancaire, chèque postal ou virement postal/Cheques are payable to:

CDR-Gauthier-Villars CCP La Source 3336861.

Les abonnements partent du premier fascicule de l'année en cours. /

Subscriptions begin with the first issue of the year.

Vente au numéro: Navarin Éditeur

31, rue de Navarin, 75009 Paris Tél. (1) 48780565

Prix de l'abonnement pour 1991 : Institutions : $390 \mathrm{~F}$ (France) et $446 \mathrm{~F}$ (Étranger)

Particuliers : $300 \mathrm{~F}$ (France) et $300 \mathrm{~F}$ (Étranger)

Prix du numéro: $90 \mathrm{~F}$ 


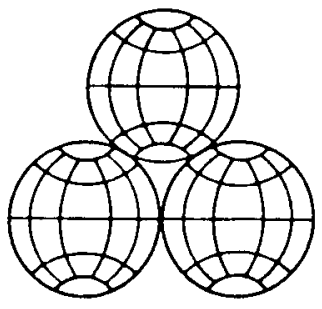

\section{REVUE TIERS MONDE}

REVUE TRIMESTRIELLE

publièe par

L'INSTTTUT D'ÉTUDE DU DÉVELOPPEMENT ÉCONOMIQUE ET SOCIAL DE L'UNIVERSITÉ DE PARIS I

$N^{\circ} 127$ - Juillet-Septembre 1991

\section{" INVESTISSEMENT-TRAVAIL " ET DÉVELOPPEMENT \\ des approches et pratiques renouvelées?}

sous la direction d'André Guichaoua

André Guichaoua : L' " investissement-travail ", le renouveau d'un concept ?

Bilan d'expériences

Gilbert Etienne : Investissement-travail et emploi en Inde

Claude Aubert : Investissement-travail et infrastructures agricoles : bilan et actualité des corvées en Chine

Daniel Martinez : Les fonds d'investissement social en Amérique centrale et au Panama André Guichaoua : Les " travaux communautaires » en Afrique centrale

Enjeux et perspectives

Jean Majeres et Steven Miller : La rémunération dans les programmes d'investissementtravail : entraide, rétribution ou salaire?

Jacques Gaude et Réjean Tremblay : De l'importance des systèmes d'information dans les programmes d'infrastructures rurales dans les pays en développement

Loic Picard : Investissement-travail ou travail forcé ?

Bibliographie thématique par Alain Léon

Mario Lanzarotti : Chocs exogènes, ajustement et changement structurel en Corée du Sud Jean Le Nay et Jean Mathis : Une méthode d'analyse de la dépendance extérieure d'un pays en développement

Germán Velasquez : Origine et évolution du concept du médicament essentiel promu par l'oms

CHRONIQUE INTERNATIONALE - BIBLIOGRAPHIE

\section{RÉDACTION ET ADMINISTRATION}

INSTITUT D'ÉTUDE DU DÉVELOPPEMENT ÉCONOMIQUE ET SOCLAL

58, boulevard Arago, 75013 Paris - Tél. : 43-36-23-55

\section{ABONNEMENTS ET VENTE}

PRESSES UNIVERSITAIRES DE FRANCE

Département des Revues : 14, avenue du Bois-de-l'Epine, BP 90, 91003 Evry Cedex

Tél. (1) 60778205 - Télécopie (1) 60792045

Télex : PUF $600474 \mathrm{~F}$ - Compte Cheques Postaux : Paris $130269 \mathrm{C}$

Abonnements annuels pour l'année 1991. - France : $370 \mathrm{~F}$; Etranger : $460 \mathrm{~F}$

Prix du présent numéro : $120 \mathrm{~F}$ 


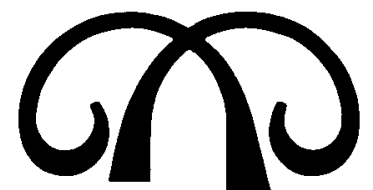

AOUTT-SEPTEMBRE 1991

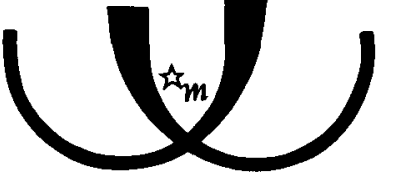

CRITIQUE

\section{BERLIN}

\section{N'EST PLUS UNE ÎLE}

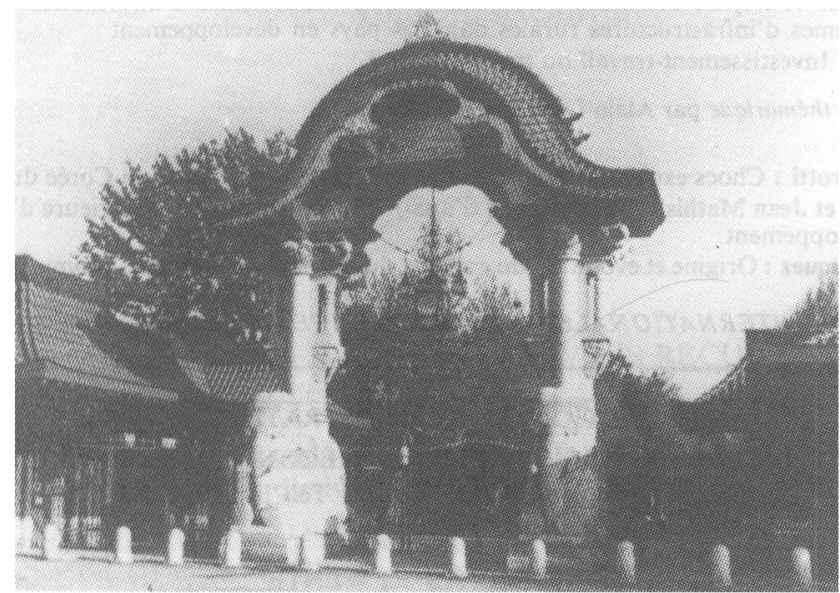

Revue générale des publications françaises et étrangères Publiée avec le concours du Centre National des Lettres 


\section{A R C H I V E S \\ E UR OPÉEN N ES D E S O C I O L O G I E}

\section{SOM M A I R E}

VITORINO MA GALHÃES GODINHO

Entre mythe et utopie : les grandes découvertes. La construction de l'espace et l'invention de l'humanité aux $X V^{e}$ et $X V I^{e}$ siècles.

D I E G O G A M B E T T A

'In the beginning was the Word...'. The symbols of the mafia .

The puzzling scope of rationality

CLAUS OFFE

Introduction.

R A L P H H T TURNER

The use and misuse of rational models in collective behavior and social psychology.

J ON ELSTER

Rationality and social norms.

M A R G ARET LEVI

Are there limits to rationality?

STEVEN L UK ES

The rationality of norms.

\section{NOTES CRITIQUES}

JEAN-Hugues DÉCHAux, Structures de parenté et sociétés paysannes : deux points de vue et des suggestions pour une sociologie de la parenté dans les sociétés urbaines MICHAEL MULKAY \& JOHN ERNST, The changing profile of social death $\rightarrow$ JEAN-Claude lamberti, Montesquieu in America.

R É D A C T IO N

Éric de Dampierre - Jon Elster Jacques Lautman - Steven Lukes -- Claus Offe 12, rue de l'École de médecine, 75270 Paris Cedex 06 CORRESPONDANTEN AMÉRIQUE Lewis A. Coser, Boston College, MA 02167) 


\section{Revue française de sociologie}

publiée avec le concours de

L'INSTITUT DE RECHERCHE SUR LES SOCIÉTÉS CONTEMPORAINES

CENTRE NATIONAL DE LA RECHERCHE SCIENTIFIQUE

59-61, rue Pouchet, 75849 Paris Cedex 17 - Tél. : 40.25.11.87 ou 88

Les rééducations à l'école primaire :

enquête d'évaluation Alain MINGAT

Les autodidactes

à l'Université de Saint-Denis Claude F.-POLIAK

Urbanisation et structures familiales

en Algérie (1948-1987) Maurice GUETTA

Culture et " effet sociétal " Philippe d'IRIBARNE

\section{LES IDÉES ET LES MOTS}

LES LIVRES

TABLES 1986-1990

Abonnements :

L'ordre et le paiement sont à adresser directement à :

Centrale des Revues, CDR

11, rue Gossin, 92543 Montrouge Cedex -. Tél. : (1) 46.56.52.66 CCP La Source 33-368-61 CDR-Gauthier-Villars

Les abonnements sont annuels et partent du premier numéro de l'année en cours.

Tarif 1992 : L'abonnement (4 numéros) France

Vente au numéro : $\quad$ Soit par correspondance auprès des :

Presses du CNRS, 20-22, rue Saint-Amand, 75015 Paris

Tél. : (1) 45.33.16.00 - Télex : $200356 \mathrm{~F}$

Soit auprès des librairies scientifiques 
ÉTUDES RURALES

$\mathrm{N}^{\circ} 118-119$

Avril-septembre 1990

\section{La météo}

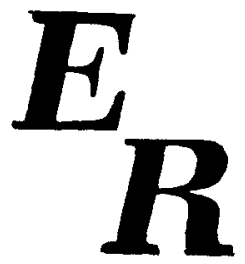

Pour une anthropologie du temps qu'il fait

M. DE LA SOUdière, Revisiter la météo

P. PRADO, Paysage après la tempête. Les retombées d'une catastrophe naturelle : ordre et désordre dans le culturel

L. CAILlET, Pluies et vents, figures du destin. Le pouvoir impérial et la maîtrise du temps au Japon

M.-F. NOËL-WALDTEUF̂EL, La météorologie entre science et savoir. L'affaire Mathieu de la Drôme

v. PELOSSE, Observation météorologique et sociétés savantes de province ou la désignation du bon objet scientifique (1821-1878)

J.-P. MARCHAND, Le climat : de l'analyse régionale au stéréotype

G. LARIVIÈRE, Le climat, question de mode ?

I. KLIS-LILIENTHAL et M. DESROZIERS, La biométéorologie humaine : deux exemples

É. DESBOIS, Soldats à découvert par temps de guerre

S.-L. LAMONTAGNE, L'hiver au Québec. Une lecture du temps qu'il fait

P. SANSOT, Jamais la météorologie n'abolira l'art d'interpréter les signes venus du ciel. Le chariot des quatre saisons à Narbonne

G. LAPOUGE, Contribution à une théorie des climats

\section{Espaces péri-urbains}

F. DUBOST, Le lotissement, implant urbain en milieu rural ?

R. BONNAIN, Les citadins aux champs

P. VIOLIER, Les exploitations agricoles face aux expropriations. L'exemple

de la communauté urbaine de Lille entre 1950 et 1980

P. CADÈNE, L'usage des espaces péri-urbains. Une géographie régionale des conflits

Chronique scientifique - Notes critiques - Comptes rendus 


\title{
RAISON PRÉSENTE
}

Directeur : Victor LEDUC

\author{
SPÉCIAL NUMÉRO 100 - 1966-1991 \\ octobre-novembre-décembre 1991
}

\section{ANS D'HISTOIRE : POLITIQUE, SCIENCES, ARTS}

$\begin{array}{ll}\text { Victor LEDUC } & \text { - Destin du léninisme } \\ \text { Thierry PFISTER } & \text { - Une décennie Mitterrand } \\ \text { Jean BOUSSINESQ } & \text { - Le pape est-il devenu économiste ? } \\ \text { Alain POLICAR } & \text { - Racisme et antiracisme } \\ \text { Françoise PICQ } & \text { - Un féminisme hexagonal } \\ \text { Christian RUBY } & \text { - Le baby-boom de l'art } \\ \text { Maurice AUDEBERT } & \text { - Vingt ans après } \\ \text { Guy BRUIT } & \text { - Retour à la Comédie Française } \\ \text { Yves GALIFRET } & \text { - Réduction et émergence } \\ \text { Gabriel GOHAU } & \text { - Sciences de la Terre en révolution } \\ \text { Michel GAUTHIER DARLEY } & \text { - Michel Foucault et la grammaire générale } \\ \text { Christian MAGNAN } & \text { - Sur le principe anthropique } \\ \text { Maurice CAVEING } & \text { - Réflexions sur le marxisme et les sciences sociales } \\ \text { Hélène DELORME } & \text { - Les politiques agricoles dans les pays industriels } \\ \text { Pierre LUTZ } & \text { - Danse avec les loups ou le péché originel américain }\end{array}$

Le numéro: 75 francs

Abonnement : 225 F. (France) 235 F. (Étranger)

Nouvelles Éditions Rationalistes

14, rue de l'École Polytechnique - 75005 Paris

Tél. 46.33.03.50 

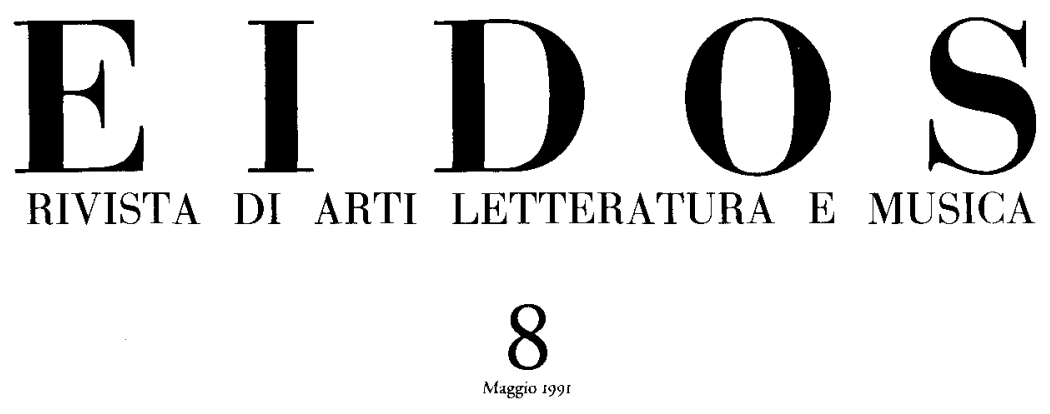

Francesco Furlan

Per una teoria del verso italiano

Ludovica Scarpa

L'architettura di Federico Guglielmo

Christian Beaufort-Spontin

Armature come abiti

Giovanni Maniscalco Basile

Mosca Terza Roma

Fabrizio Borin

Tarkovskij e il volo

Alexandre Doroszlaï

La cartografia dell'Ariosto

Gabriele Foccardi

Guida di viaggio al Lago Occidentale

ASOLO ARTI

Direttore Massimo Scolari

Collegio di direzione. Carlo Bertelli, Sandro Briosí,

Donatella Calabi, Daniele Del Giudice, Valerio Marchetri,

Giovanni Morelli Redazione: via Regina Cornaro, 224

C.P. 25 - 3 IOII Asolo
Distribuzione e abbonamenti: Elenond s.r.l. via Trentacoste, 7 - 20134 Milano

Prezzo di una copiat 1, 20,000 Esteros. 1. 25.000 Arretrati: L. 20.000 Abbonamento per tre numert. Italia I., 50.000 Estero L. 70.000 


\section{PROMETEO}

\section{Mondadori Business Information Rivista trimestrale di scienze e storia Direttore scientifico: Valerio Castronovo}

Comitato scientifico: M. Augé, M. Aymard, J. Beck, D. Bourt, P. Burke, A. Carotenuto, V. Castronoto, $N$. Cbomsky, A Dancbin, M. Detienne, U. Eco, I. Eibl-Eibesfeldt, P.K Feyerabend, L Gambi, F.M. Gil, G. Gionello, .M. Godelier, H.E. Gombrich, J. Goody, F. Héritier, A Hirschman, G. Holton, A Jacquand, J. Kocka, J.D. Laiowx, V. Lantemari, J. Le Goff, R.C. Lewontin, N. Lubmann, C. Magris, G.L Mosse, W.H. Newton-Smith, A Olinerio, A Piatigorsky, C. Poni, T. Regge, J. Revel, I. Sacbs, G.W. Stocking jr., V. Strada, P. Sylos Labini, K Thomas, V. Valeri, N. Wachtel.

\section{Anno IX n. 34}

Giugno 1991

Aldo Carotenuto: Metamorfosi dell'anima

Ignacy Sachs: La grande mutazione

Sergio Ribichini: Gli dei di Annibale

Hanna Dziechcinska: Le parole del corpo

Carlo Carboni: Cittadinanza

Valerio Valeri: Polifonia del sapere

Piero Racanicchi: Sui monti di Tolomeo

Paolo Prato/Gianluca Trivero: La fine dell'aventura

Silvia M.S. Carvalho: Cacciatori e raccoglitori

Jonathan Jacky: Pazicnti a riscbio

Francesco Ghiretti: Forma e funzione

Note e rassegne:

Pro e contro: $K$ Sbrader-Frechette/Cesare Marchetti - Ambiente e tecnologia L'immaginario matematico: Berkelius - Ars combinatoria

Tecnologie: Frank Forecaster - L'evoluzione continua

Tesi e ipotesi: Marta Petricioli - Archeologia politica

L'angolo della memoria: C.P.N. Stochos - Quest'acqua cosi inuadente

Frammenti di Esculapio: Maria Baiocchi/Stefano Cagliano - Eppur si vede...

Celebri ignoti: Gabriella Lelli - La scienziata "santa"

Variazioni: Viva Tedesco - Divagazioni sulle barbe

Cosmologie: Angioletta Coradini - Due navicelle verso Fobo.

Lordine va inviato insieme al pagamento a:

Amoldo Mondadori Editone Servizio Abbonamenti 20090 - Segrate (MI) Italia

Tariffe valide per il 1990 


\section{techniques Goculture}

REVUE SEMESTRIELLE

Comité de patronnage

Lucien Bernot, Andre-G. Haudricourt André Leroi-Gourhan $\dagger$, Axel Steensberg

Secrétaire général Robert Cresswell

\section{Numéro 16 juillet-décembre 1990 \\ Des machines et des hommes}

\author{
M. Akrich \\ T. Poujol \\ J. Broustail \\ V. Rabeharisoa \\ M. Akrich \\ C. Chestier \\ C. Méadel \\ J. Bourdon \\ B. Pfaffenberger
}

\section{Présentation}

L'émergence du réseau d'assainissement pneumatique

La destinée paradoxale de l'automatisme

Mesures "techniques", Mesures "morales"

De la sociologie des techniques à une sociologie des usages

Des techniques en quête d'artistes, ou les complexes articulations entre l'art et la science

Les images sonores, naissance du théâtre radiophonique

Film ou vidéo, réflexions sur la pérennité d'une controverse technique

The Hindu Temple as a machine, or the Western Machine as a Temple
$\mathrm{N}^{\circ} \mathrm{s} 1$ et 2, 1983

$\mathrm{N}^{\circ} 3$ janv.-juin 1984 $N^{\circ} 4$ juil.-déc. 1984

$N^{\circ} s 5$ et 61985

$\mathrm{N}^{\circ} 7$ janv.-juin 1986

$\mathrm{N}^{\circ} 8$ juil.-déc. 1986

$\mathrm{N}^{\circ} 9$ janv.-juin 1987

$\mathrm{N}^{\circ} 10$ juil.-déc. 1987

$\mathrm{N}^{\circ} 11$ janv.-juin 1988

$\mathrm{N}^{\circ} 12$ juil.-déc. 1988

$\mathrm{N}^{\circ} 13$ janv.-juin 1989

$N^{\circ} 14$ juil.-déc. 1989

$\mathrm{N}^{\circ} 15$ janv.-juin 1990
Actes de la table ronde "technologie culturelle"(1982)

Des choses dont la recherche est laborieuse"

Aspects des agricultures insolites de l'Amérique indienne

Les langages du vêtement

Par où passe la technologie I et II

De l'Himalaya au Haut Atlas, de l'Asir aux Andes

Techniques moyen-orientales

Des idées pour observer

D'autres idées pour observer

Persistances et innovations

Symboles et procès techniques

Corpus

Inde

Du soufflé à la forge

\begin{tabular}{lll} 
& \multicolumn{2}{c}{ CONDITIONS DE VENTE } \\
\hline \multirow{2}{*}{ Abonnement } & 1991 \\
& $\begin{array}{l}\text { Particulier } \\
150 \mathrm{~F}\end{array}$ & $\begin{array}{l}\text { Institution } \\
180 \mathrm{~F}\end{array}$ \\
Etranger & $150 \mathrm{~F}$ & $180 \mathrm{~F}$
\end{tabular}

vente au numéro - prix au numéro $75 \mathrm{~F}$

S'adresser au 


\title{
REVUE DE L'HISTOIRE DES RELIGIONS
}

Publication trimestrielle fondée en 1880

\author{
COMITÉ DE PATRONAGE \\ André BAREAU, André CAQUOT \\ Jean DELUMEAU, Jean-Pierre VERNANT \\ DIRECTION \\ Antoine GUILLAUMONT, Charles AMIEL \\ CONSEIL DE RÉDACTION
}

Gabriel AUDISIO, Jean BAZIN, Jacques LE BRUN

Nicole LORAUX, Charles MALAMOUD, Maurice OLENDER

Au sommaire des fascicules 2 et 3 de 1991

\section{HISTOIRE DES RELIGIONS ET COMPARATISME : \\ LA QUESTION INDO-EUROPÉENNE}

Présentation (C. Malamoud). - Contribution à une épistémologie dumézilienne : l'idéologie (D. Dubuisson). - Some Gods of Pre-Islamic Nuristan (N. J. Allen). - Réalité des Indo-Européens : les diverses apories du modèle arborescent (J.-P. Demoule). - Hypothèse indoeuropéenne et modes de comparaison (G. Charachidzé).

L'expérience du Prophète Elie : qol d mama daqqa (M. Masson). Jason ou le retour du pécheur. Esquisse de mythologie argonautique (B. Mezzadri). - Baldassare Labanca en France (A.-C. Faitrop-Porta).

Comptes rendus, Notes bibliographiques

Chroniques, Livres reçus.

\author{
CORRESPONDANCE \\ Revue de l'Histoire des Rellgions \\ PUF, 12 rue Jean-de -Beauvais, 75005 PARIS \\ VENTE ET ABONNEMENTS \\ Presses Universitaires de France \\ 14, avenue du Bois-de-l'Epine \\ B. P. 90, 91003 Evry Cedex \\ (C.C.P. : PARIS 1302-69 C) \\ Abonnement 1991 (4 fascicules) \\ France : $315 \mathrm{~F}$ Etranger : 400F \\ Prix au numéro : $105 \mathrm{~F}$
}




\section{EUROPEAN JOURNAL OF COMMUNICATION \\ RECENT CONTENTS \\ Editorial \\ The Internationalization of Television R. Negrine and S. \\ Papathanassopoulos \\ The Role of the Alternative Press in the Agenda-building Process: Spill-over Effects and Media Opinion Leadership Rainer Mathes and Barbara Pfetsch \\ The Contagiousness of Mass-mediated Terrorism \\ Hans-Bernd Brosius and Gabriel Welmann \\ Knowledge Gaps Revisited: Secondary Analyses from Germany Reinhold Horstmann \\ Feature: \\ Public Television in Crisis: Critiques Compared in Norway and Britain Trine Syvertsen \\ Revlews \\ Abstracts

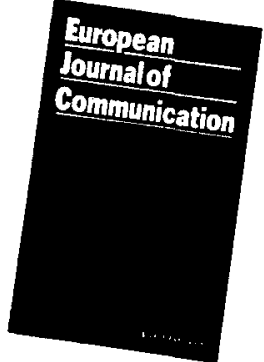 \\ Edited by Jay G. Blumler, Denis McQuail and Karl Erik Rosengren \\ ...the commitment of the EJCs editors, in the spirit of academic freedom, to opening the pages of the joumal to contentious and provocative material is palpable.' Times Higher \\ Education Supplement \\ Published quarterly in March, June, September and December}

\section{Make sure that you subscribe now!}

\section{Use the order form below and save $20 \%$}

\section{0\% Discount Order Form}

Send this order form to:

\section{Sage Publications}

6 Bonhill Street, London EC2A 4PU, UK Tel: 071-3740645

Or why not fax us your order on

071-3748741?

US Orders to:

PO Box 5096, Newbury Park, CA 91359, USA

Y Yes! I want to subscribe to European Journal of Communication at a $20 \%$ Discount

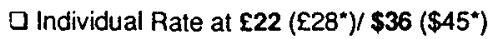

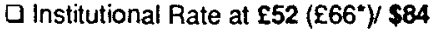

(\$106*) Usual 1991 rate

Name

Address
Signature

Date

\section{THREE WAYS TO PAY!}

a CHEQUE!... I enclose a cheque (made payable to Sage Publications)

口 GIRO!... I have today paid by International Giro to A/c No 5480353

Date

口 CREDIT CARD!... Please charge my credit card

$\square$ Mastercard $\square$ Access $\square$ Visa

- Barclaycard O American Express

$\square$ Diner's Club $\square$ Eurocard

Card Number

Expiry Date

$\longrightarrow$ 


\title{
Bringing you critical scholarship on modern Europe from a social, intellectual, political, and economic perspective
}

\section{THE JOURNAL OF MODERN HISTORY}

\begin{abstract}
A Broad Scope: European History from 1500 to the Present Founded in 1929, The Journal of Modern History is recognized as the leading American journal for the study of European intellectual, political, and cultural history. The Journal's geographical and temporal sweep-the history of the European continent, including Russia and the Balkans, since the Renaissance-makes it unique in scope.
\end{abstract}

\section{A Diversity of Approaches: Primary Research, Historiographical Essays, and Book Reviews \\ Combining both "traditional" and "innovative" ways of writing history, the JMH presents a diversity of scholarly perspectives and orientations and assesses trends and developments in historiography. As the premier reviewing journal for European history, it will save you time and money in your search for relevant books.}

\section{An Occasional Special Issue or Supplement:}

JMH devotes special issues and supplements to important examinations or re-examinations of crucial topics such as: Imperial Russian and Soviet History (1990), French Politics in 1788 (1988), National Socialism (1987), Politics and Society in the Holy Roman Empire (1986), and Political Practice in the French Revolution (1984).

Edited by John W. Boyer and Julius Kirshner

Published quarterly by The University of Chicago Press

Regular one-year subscription rates: Individuals \$28.50; AHA Individual Members \$21.50; HA Individual Members (Great Britain) $\$ 21.50$; Students (with copy of ID) $\$ 21.50$; Institutions $\$ 57.00$. Outside USA add $\$ 6.00$ for postage. Visa and MasterCard accepted. To order send complete credit card information or payment in U.S. dollars to The University of Chicago Press, Journals Division, Dept. SS1SA, P.O. Box 37005, Chicago, IL 60637. 


\section{Ethnologie française}

\section{Entre l'oral et l'écrit $3 / 1990$}

Marc Soriano

Entre l'oral et l'écrit

Georges Mounin

Oral/Ecrit : préliminaires linguistiques

Bernard Lahire

Sociologie des pratiques d'écriture

Nicole Fabre

Du stade oral à l'oralité

Fausta Garavini

Montaigne entre l'écrit et l'oral

Catherine Clément

Le «Créchois»

Béatrice Didier

La voix de Mère-grand
Clara Gallini

Denis Laborde

"Tout raccorder et tomber juste".

l'art $d u$ bertsulari basque

Danielle Alexandre-Bidon

Ecrire le son au Moyen Age

Louis Assier-Andrieu

Le droit symbolique ou les pièges de l'altérité

Jean-Pierre Peter

Quand les paroles s'envolent et qu'd terre l'écrit reste

Philippe Richard

Mesurer le non-mesurable : le cas des proverbes

\section{Paradoxes de la couleur $4 / 1990$}

Marie-Christine Pouchelle

Paradoxes de la couleur

Michel Pastoureau

Une histoire des couleurs est-elle possible?

Claude Gaignebet

Le sang-dragon du Jardin des Délices

Maurice Agulhon

Les couleurs dans la politique française

Michel Pigenet

Le vocabulaire des couleurs

dans l'identité politique et sociale

Jean-Luc Bonniol

La couleur des hommes

comme principe d'organisation sociale
Sylviane Leprun

Exotisme et couleur

Martin de la Soudière

Les couleurs de la neige

Notes de recherche

Sylvie Treille

Colorations sur photographie:

la photopeinture

Henriette Touillier-Feyrabend

Les couleurs du prestige en publicité

Martine Jaoul

Dans les coulisses de la mode:

le métier de teinturier

Rédaction : Musé national des Arts et Traditions populaires

6, av. du Mahatma-Gandhi - 75116 Paris - Tel. : (1) 40.67 .90 .00

Abonnements (4 numeros par an) : Armand Colin Edicur

Boîte postale $22-41353$ Vincuil Códex

Vente au numéro : par lintermediaire de votre libraire habitucl ou su comptoir-librairie du Musce national des Arts et Traditions populaires.

Prix de l'abonnement pour 1991 (les abonnements partent du premier fascicule de l'annce en cours) :

Institutions : $550 \mathrm{~F}$ (France) - 135 \$ US (Etranger)

Particuliers : 412 F (France) - $100 \$$ US (Etranger)

Prix au numero: $115 \mathrm{~F}$ 


\title{
HISTOIRE MESURE
}

\author{
1991 \\ Volume VI - ${ }^{\circ} 1 / 2$ \\ SERIES TEMPORELLES
}

\section{Sommaire}

Editorial

\section{LES METHODES}

Pierre-Alain JAYET, Quelques notions sur l'analyse spectrales des séries temporelles

Bertrand ROEHNER, La liaison entre les conjonctures de prix des produits de base. Le cas de l'Allemagne au XIXe siècle

\section{ETUDES DE CAS}

Jean-Yves GRENIER et Alexandre MATHIS, Séries temporelles, structure et conjoncture : le prix du blé à l'époque moderne

Alain GUERREAU, Observations statistiques élémentaires sur une série de dates de vendanges (fin XVe - fin XXe siècle)

Jean-Michel CHEVET et Pascal SAINT-AMOUR, L'intégration des marchés du blé en France au XIXe siècle

Daniel ZAJDENWEBER, Chronique d'un randonneur centenaire : le Dow Jones

\section{LES DOMAINES}

Noẹl BONNEUIL, Temporalités en démographie historique

André SALEM, Les séries textuelles chronologiques

\section{REFLEXIONS CRITIQUES}

Jean-Yves GRENIER, Réflexions libres sur l'usage des méthodes statistiques en histoire

\section{NOTES ET DISCUSSIONS}

Les livres (compte-rendu par G. Depeyrot, H. Ducasse, J.-Y. Grenier, J. Heffer, M.-A. Polo de Beaulieu)

Résumés des articles

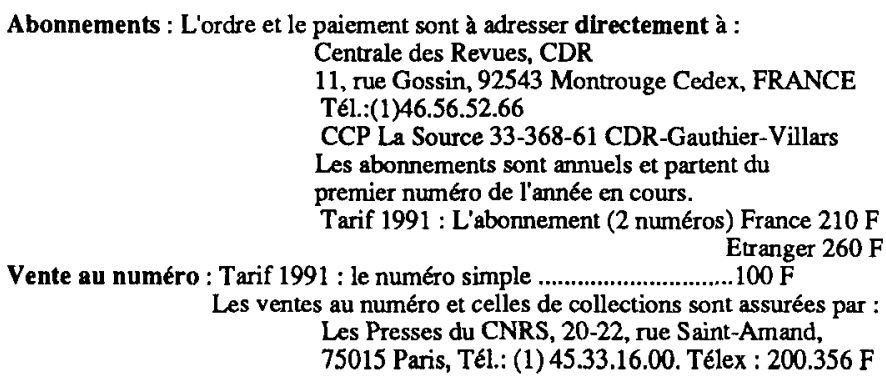




\section{Revue de la Bibliothèque Nationale}

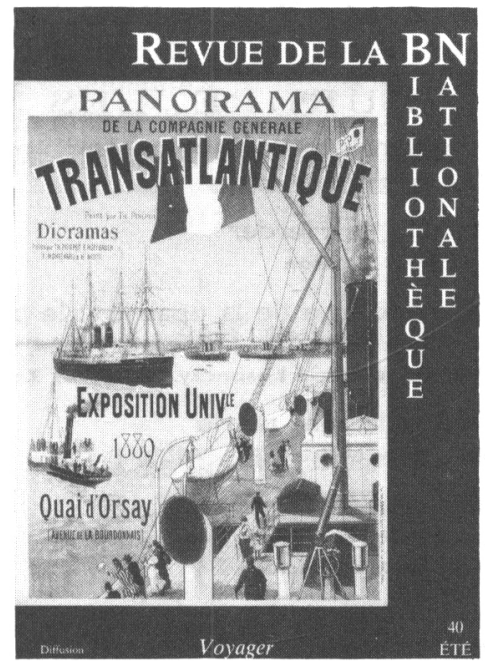

Directeur de la publication : Emmanuel Le Roy Ladurie

Rédacteur en chef :

Antoinette Fauve-Chamoux

Conseillers de la rédaction : François Lafon,

Jacqueline Sanson

Secrétaire de rédaction : Marie-Claire Saint-Germier

Rédaction : RBN

58, rue de Richelieu

75084 Paris Cedex 02

Tel. 47038107

Périodicité : Trimestrielle ISSN $0249-7344$

Format : $21 \times 29,7$

nombreuses illustrations noir et couleur, 88 pages

Diffusion : Armand Colin
Reflet de toutes les activités de la Bibliothèque Nationale, la Revue de la $B N$ s'adresse à un vaste public intéressé aussi bien par la politique de conservation et de diffusion du patrimoine culturel national sous toutes ses formes ( manuscrite, imprimée, photo, disque, vidéo, informatique, etc. ) que par les multiples travaux et recherches entrepris sur ses fonds. Une chronique illustrée des enrichissements des collections par dons ou achats dans les divers départements de la Bibliothèque Nationale est complétée par la présentation des nombreuses expositions - une dizaine chaque année - qui se tiennent dans l'établissement, rue Richelieu, galerie Colbert, a l'Arsenal ou dans les antennes de province.

La revue signale publications, catalogues et toute activité liée à la vie de l'établissement, en privilégiant les aspects originaux et spécifiques de la recherche française sur le plan international.

Numéros à thèmes.

Tarifs 1992

\begin{tabular}{|c|c|c|}
\hline Abonnements & France & Elranger \\
\hline Particuliers & $278 \mathrm{~F}$ & 82 \$ US \\
\hline Société des Amis de la & & \\
\hline Bibliothèque Nationale & & $180 \mathrm{~F}$ \\
\hline Règlement à l'ordre de: & $\begin{array}{r}A \\
41353\end{array}$ & $\begin{array}{l}\text { and Colin } \\
\text {.P. } 22 \\
\text { neull Cedex } \\
\text { rance }\end{array}$ \\
\hline
\end{tabular}

Vente au numéro : $94 \mathrm{~F}$

chez votre libraire ou Librairie Colbert 6 rue des Petits-Champs - 2 rue Vivienne 75002 Paris - Tel : 47038571

Armand Colin 103, bd Saint-Michel 75240 Paris Cedex 05

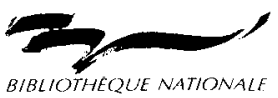




\section{CARNETS DU PATRIMOINE ETHNOLOGIQUE}

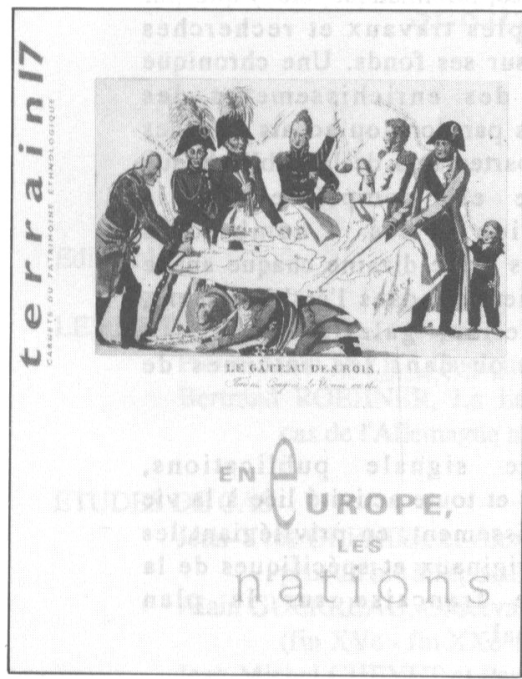

Mission du Patrimoine ethnologique

65 , rue de $R$ ichelieu, 75002 Paris

Tél. (1) 40158527

Conseil de rédaction: Gérard Althabe

Christian Bromberger ФैClaudine Fabre-Vassas Bertrand Hell ज़Gérard Lenciud $\sum_{\bar{D}}^{\bar{D}}$

$\mathrm{S}_{\mathrm{N}^{\circ}} 2$ : Anthropologie industrielle

奇 $N^{\circ} 3$ : Ethnologie urbaine

№ 4 : Famille et parenté

ปั้ $N^{\circ} 5$ : Identité culturelle et خappartenance régionale

NN 6 : Les hommes ct le milieu naturel

$\mathrm{N}^{0} 7$ : Approches des communautés ज्ञ étrangères en France

글 $N^{\circ} 8$ : Rituels contemporains

$\mathrm{N}^{\circ} 9$ : Habiter la maison

${ }_{\infty}^{\infty} N^{0} 10$ : Des hommes et des bêtes

$\mathrm{N}^{\circ} 11$ : Mélanges

NN 12 : Du congélateur

Sau déménagement

$\mathcal{N}_{\text {(consommation familiale) }}$

○े $N^{0} 13$ : Boire

$N^{\circ} 14$ : L'incroyable et ses preuves

$N^{\circ} 15$ : Paraitre en public

$N^{0} 16$ : Savoir-faire
Terrain paraît semestriellement. Son ambition est de faire connaître les études ethnologiques du domaine français, notamment celles réalisées avec le concours de la mission du Patrimoine ethnologique. La revue accueille également des articles d'ethnologie européenne.

Mais Terrain est aussi une tribune offerte à ceux qui souhaitent faire part de leurs réalisations (muséographiques, audiovisuelles, culturelles...), rendre compte de leurs travaux en cours, échanger librement informations et expériences, engager le débat: les rubriques «Repères» et «Infos» leur sont destinées.

Nous comptons donc sur votre participation qui nous aidera à faire de ces pages un instrument utile.

Revue semestrielle. Format $210 \times 270 \mathrm{~mm}$. 176 pages. Vente au numéro: prix $70 \mathrm{~F}(+23 \mathrm{~F}$ de frais de port $)$ à adresser au

CID, 131, bd Saint-Michel, 75005 Paris.

Tél. : (1) 43.54.47.15

Les abonnements doivent être adressés à la CNMHS, 62, rue Saint-Antoine, 75004 Paris.

$280 \mathrm{~F}$ pour 4 numéros sur 2 ans.

\section{sommaire}

EN EUROPE, LES NATIONS

Le nationalisme en apesanteur

Ernest Gellner

Lumière de Herder

Max Caisson

Anthropologie de la «patrie»: le patriotisme hongrois

- Anne-Marie Losonczy et András Zempleni

Un peuple sans patrie

Michael Stewart

L'universel et le relatif

A propos d'un ouvrage de Tzvetan Todorov

Gérard Lenclud

Un moment de la conscience européenne Michel Izard

«Un clip vaut Shakespeare»

Entretien avec Alain Finkielkraut

Jeanne Favret-Saada et Gérard Lenclud

Nationalité et nationalisme en Roumanie

- Vintila Mihăilescu

«Eminemment français». Nationalisme et musique Jacques Cheyronnaud

Mises en scène du racisme italien

Clara Gallini

\section{REP È ES}

"Où la terre s'achève et la mer commence»

Une anthropologie du Portugal

Jean-Yves Durand

Ethnologie et muséologie dans un milieu insulaire, les Canaries

Entretien avec Rui de Sousa Martins

François Hubert

L'architecture rurale des deux côtés de la Manche François Calame

Regards ethnologiques sur l'Europe

Thomas K. Schippers

T R A V A U X

I N F OS 


\title{
Sciences Sociales et Santé
}

Vol. IX $n^{\circ} 3$ Septembre 1991 Revue trimestrielle

Sciences Sociales et Santé s'attache à organiser un débat entre les disciplines de sciences humaines (anthropologie, sociologie, histoire, psychanalyse, économie) dans le champ socio-sanitaire. La revue se propose de faire connaître les travaux et réflexions que ce champ suscite à l'intention de la communauté scientifique. Elle est publiée avec l'aide du Centre National de la Recherche Scientifique (CNRS), de l'Institut National de la Santé et de la Recherche Médicale et du Ministère de la Recherche et de la Technologie.

\author{
Sommaire \\ Larry L. Palmer Implications légales et morales de la \\ construction sociale de la santé : le \\ syndrome d'alcoolisme fotal aux Etats-Unis \\ Philippe Dehaene \\ Boire et avoir un enfant (commentaire) \\ Maurice Titran L'intervention médico-sociale précoce dans \\ le cadre de l'alcoolisme fœtal. L'expérience \\ du centre de Roubaix (commentaire) \\ Gilbert Duval La mort à l'île de la Réunion. \\ Réflexions sur la confrontation \\ des formes culturelles du mourir \\ Christiane Bougerol \\ Remarques sur l'échange entre les vivants \\ et les morts (commentaire) \\ Pascal Cathébras Du «burn out » au « syndrome des yuppies » : \\ deux avatars modernes de la fatigue \\ Antoine Laville Explorer le travail (commentaire) \\ Notes de lectures \\ Livres reçus \\ Résumés (anglais, espagnol)
}

\section{TARIFS D'ABONNEMENT 1991 (4 numéros par an)}

$\begin{array}{lcc} & \text { France } & \text { Etranger } \\ \text { Etudiants } & 210 \mathrm{FF} & 250 \mathrm{FF} \\ \text { Particuliers } & 280 \mathrm{FF} & 320 \mathrm{FF} \\ \text { Institutions } & 420 \mathrm{FF} & 450 \mathrm{FF}\end{array}$

Prix de vente au numéro : $110 \mathrm{FF}$

Les abonnements sont annuels et partent du premier numéro de l'année en cours. Le paiement est à effectuer à l'ordre de CDR et doit être adressé à :

John Libbey Eurotext, 6 rue Blanche, 92120 Montrouge, France 


\section{Continuity and Change}

A journal of social structure, law and demography in past societies

Continuity and Change examines the basic organisations of family, law, economy, culture and government through current research and theory in a manner intelligible to non-specialists and academics alike.

Emphasis is upon studies that draw on more than one of the traditional fields such as history, sociology, law, demography, economics or anthropology. The journal is committed to comparative studies over a broad range of cultures and time spans. It provides a balance between quantitative and qualitative data from the contributing disciplines, promoting greater dialogue and discussion among researchers and making their work accessible to awider audience.
Continuity and Change offers:

Special thematic issues

Articles on all aspects of social history

Abstracts of all articles in English, French and German

A wide range of international authors

Book reviews and review articles

\section{Subscription}

Continuity and Change is published three times a yearin May, August and December. Volume 7 (1992) is $£ 27$ for individuals; E51 for institutions; airmail is $\mathbf{£ 9 . 5 0}$ per year extra ISSN $0268-4760$

Take a closer look for free!

Please enter my subscription to Continuity and Change, Volume 7 (1992)

$\square £ 27$ for individuals; $\square £ 51$ for institutions

$\square$ Please send by airmail at $£ 9.50$ per year extra

$\square$ I enclose a cheque (made payable to Cambridge University Press and drawn against a UK bank)

$\square$ Please send me an invoice

$\square$ I have not decided, please send me a free sample copy

Name

Address

Send your coupon to: Journals Marketing Department, Cambridge University Press,

FREEPOST*, The Edinburgh Building, Cambridge CB2 1BR, England TEL: (0223)

325806 * no postage stamp necessary if posted in UK

In US \& Canada, write to Cambridge University Press, 40 West 20th Street, New York, NY 10011-4211, USA

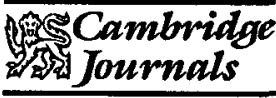




\section{CONGRÈS ET COLLOQUES}

Colloque " Robespierre " Arras 1993. De la nation artésienne à la République et aux nations organisé les 8, 9 et 10 avril 1993 par le Centre d'Histoire de la région du Nord et de l'Europe du Nord-Ouest.

- Informations : Propositions à adresser pour le 15 février 1992 au Centre d'Histoire de la Région du Nord et de l'Europe du Nord-Ouest (Robespierre, Arras 93), Université Charles de Gaulle, Lille III, BP 149.

The symposium for 1992 : "Ethonogenesis : a frontier phenomenon " on March 7 , 1992.

- Informations : Call for papers. Please write to Dr. David H. Miller, Department of History, University of Oklahoma, Norman, OK 73019.

Les XIIIe Journées d'Histoire du Droit médiéval auront lieu à Limoges dans la semaine du 23 au 27 mars 1992.

- Informations : Pierre Braun, professeur à la Faculté de Droit et des Sciences économiques, 87031 Limoges Cedex.

Seventh Congress of the European Economic Association, August 29-August 31, 1992, Dublin, Ireland.

- Informations : Call for papers. Professor John Vickers, EEA Congress Programme Chairman, University of Oxford, Institute of Economics and Statistics, St Cross Building, Manor Road, Oxford 0X1 3UL (U.K.).

Les mouvements de longue durée dans la pensée économique, colloque organisé à Montpellier en septembre 1992 par le Centre Régional de la Productivité et des Études Économiques.

- Informations : Appel à communications : Jean-Louis Escudier (CRPEE) et Marc Penin (Ass. Ch. Gide). Tél. 67.61.54.63. Secrétariat du colloque "Cycles longs ", 39, rue de l'Université, 34060 Montpellier cedex.

La fondazione biblioteca archivio Luigi Micheletti, organise un colloque sur la collaboration en Europe (1939-1941) qui se tiendra entre l'automne 1992 et le printemps 1993.

- Informations : pour tout renseignement s'adresser à la Fondazione Luigi Micheletti, via Cairoli n 9, 25122 BRESCIA, tél. 030/48578 - FAX 030/45203.

Colloque " Forêt, armées et guerres ", Groupe d'Histoire des Forêts Françaises, Paris 8, 9 et 10 octobre 1992.

- Informations : appel à communications à adresser à Andrée Corvol, Aline Fernandez, ENS, 45, rue d'Ulm, 75005 Paris (tél. 16.43.29.62.65 ou Jean-Paul Amat, Commission française d'histoire militaire, Université de Paris XII, av. du général de Gaulle, 94010 Créteil cedex (tél. 16.48.98.91.44 - p. 23-26). 


\section{CAHIERS DES ANNALES}

En vente chez ARMAND COLIN Éditeurs, 103, boulevard Saint-Michel, 75240 PARIS Cedex 5

M. BLOCH, Selgneurie française et manoir anglais, $2^{\mathrm{E}}$ édition.

M. AGULHON, Le cercle dans la France bourgeoise (1810-1848).

P. CHAUNU, Histoire quantitative, histoire sérielle.

W. G. L. RANDLES, De la terre plate au globe terrestre.

S. L. KAPLAN, Le complot de famine : histoite d'une rumeur au XVIIle siècle.

R. HALÉVI, Les loges maçonniques dans la France d'Ancien Régime.

R. E. GIESEY, Cérémonial et puissance souveraine. France, XVe-XVIle siècles.

B. MÜLLER, Bibliographie des travaux de Lucien Febvre.

La revue ANNALES ESC est disponible et peut être commandée chez les libraires suivants :

Aix-en-Provence (Librairie de I'Université); Besançon (Camponovo): Bordeaux (Mollat); Caen (J.-C. Marie) ; Dijon (Librairie de I'Université) ; Grenoble (Arthaud, FNAC, Librairie de I'Université) : Lille (Meura, Furet du Nord) ; Lyon (Flammarion, Nouveautés, La Proue) ; Marseille (Maupetit) ; Montpellier (Brèche, Sauramps) ; Nantes (Vent d'Ouest) ; Nice (FNAC) ; Paris (Librairie du Regard, Interférences, La Procure, Lavocat, Escalier, Un Moment, Picard, Temps retrouvé, Autrement dit, Boutique des Cahiers, SNC Cluny Sorbonne, FNAC Montparnasse, P.U.F., FNAC Forum) ; Pau (Tonnet) ; Poitiers (Librairie de I’Université) ; Strasbourg (Berger Levrault, Librairie des Facultés, FNAC, Kléber) ; Toulouse (Librairie des Facultés, Privat) ; Tours (Boîtes à Livres)

Pour les années 1929 à 1978 ainsi que pour les index analytiques 1929-1948 et 1949-1968, s'adresser à KRAUS REPRINT, Route 100, Millwood, New York 10546, USA. 


\section{ÉCOLE DES HAUTES ÉTUDES EN SCIENCES SOCIALES \\ CAHIERS DES ANNALES 42}

\section{bibliographie des travaux \\ de}

Lucien Febvre

\section{établie par Bertrand Müller}

Par son ampleur et sa diversité, l'œuvre de Lucien Febvre est exceptionnelle. Elle occupe une place centrale dans l'innovation historiographique de ce siècle. Pourtant elle reste de nos jours en grande partie mal connue; en particulier, elle n'a jamais fait l'objet, jusqu'aujourd'hui, d'un inventaire systématique.

Ce cahier entend précisément comblercette lacune paradoxale en proposant à l'historien et au chercheur le répertoire des écrits publiés par Lucien Febvre pendant plus d'un demi-siècle entièrement consacré au service de I'histoire. Rassemblant plus de deux mille titres, la bibliographie proposée ici témoigne de la prolixité de l'écrivain ; elle rend compte surtout des interventions multiformes de l'historien dans ses combats pour une nouvelle histoire.

Ce livre vise un objectif essentiellement pratique : complété par des index (auteurs recensés, analytique, revues), il met à la disposition des chercheurs, à côté des Tables des Annales et de la bibliographie des travaux de Marc Bloch, un nouvel instrument de travail qui devrait faciliter et encourager les recherches, trop peu nombreuses encore, sur l'œuvre de Lucien Febvre, sur l'histoire des Annales et, plus largement, sur le développement des sciences sociales contemporaines.

L'auteur a suivi des études de sociologie et d'histoire à la Faculté des Sciences Sociales et Politiques de l'Université de Lausanne et à l'École des Hautes Études en Sciences Sociales. Assistant d'historiographie et de méthodologie historique à Lausanne, il poursuit une recherche sur les recensions critiques de Lucien Febvre et sur l'historiographie française au XIX ${ }^{\bullet}$ siècle.

1990. 254 pages. $95 \mathrm{~F}$.
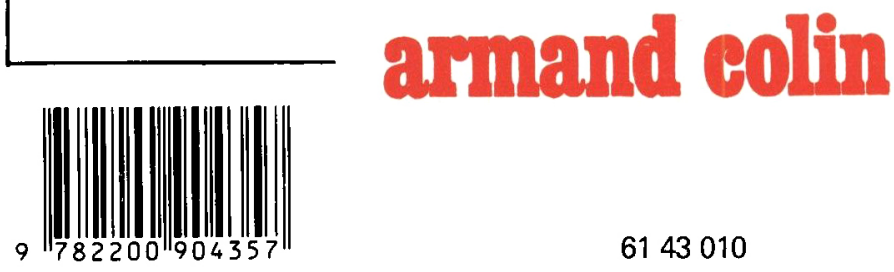